\title{
Noise propagation in feedback coupling between cell growth and metabolic activity
}

\author{
A. Borri P. Palumbo A. Singh
}

\begin{abstract}
Size control is usually exerted in living cells by properly sensing the external inputs (like nutrients) and, accordingly, by activating the metabolic pathways in order to set and adjust their own growth rate. In this framework, experimental results have recently highlighted the role of metabolic noise, usually neglected because of its averaging over the great deal of reactions involved in metabolic networks. In this note, a basic model of the interplay among metabolic enzymes activity, resource allocation and growth rate is introduced. A noise source is supposed to affect the enzymatic activity. The model includes a feedforward action of the resource on the enzyme dynamics (modulated by growth), as well as a feedback of the enzyme on the resource production rate. A Stochastic Hybrid System formulation is exploited to investigate how the noise propagates through the metabolic pathway. Model-based results support the hypothesis that fluctuations in the enzyme production perturb cellular growth, and not vice versa, because of an apparent delay in the cross-correlation function. This result is coherent with single-cell recent experimental results.
\end{abstract}

Index Terms-Metabolic pathways, Enzymatic Reactions, Systems Biology, Feedback/Feedforward loops

\section{INTRODUCTION}

The comprehension of how complex biological functions emerge from the genomic structure of a living cell, modulated by environmental cues, is an open challenge for systems biology, and computational models are expected to increase the understanding on cellular emergent properties, that arise from the interactions of a large numbers of gene products and metabolic reactions. What make these phenomena trickier to investigate are the many feedback and feedforward mechanisms linking gene expression to metabolism and vice versa. For instance, the environment (mostly the nutrient availability) strongly influences the growth rate of many organisms (spanning from bacteria to eukaryotic cells), and the growth rate is known to characterize the metabolism of the cell, eventually leading to different cell size and macromolecular resource allocation with the aim of complying with specific fates and developmental programs. Indeed, growth-dependent molecular players control the cellular resource allocation, for instance facilitating or inhibiting enzymatic reactions or modulating the correct gene expression, and a different resource allocation influences in feedback the growth rate of the cell thus controlling, by means of growth rate, in an

A. Borri, and P. Palumbo are with the Istituto di Analisi dei Sistemi e Informatica "A. Ruberti", Italian National Research Council (IASI-CNR), Via dei Taurini, Roma, Italy. Email addresses: \{alessandro.borri, pasquale.palumbo\}eiasi.cnr.it

A. Singh is with Department of Electrical and Computer Engineering, Biomedical Engineering, Mathematical Sciences, Center for Bioinformatics and Computational Biology, University of Delaware, Newark, DE USA 19716. E-Mail address: absingh@udel.edu indirect way, the metabolic enzymes activity (see, among the others, [2], [1], [6], [14], [13], [12]). Within this framework, molecular stochasticity has been usually related to enzymatic reactions or gene expression, assuming that these random fluctuations do not propagate at a metabolic level, and could be averaged and substantially neglected because of their averaging over the great deal of reactions involved in metabolic networks. Instead, recent single-cell investigations have highlighted how fluctuations in gene expression and enzymes may affect metabolic fluxes as well as the rate of cellular growth [11], [23], [15].

This note investigates noise propagation for a basic model dealing with the tight interplay between resource allocation and growth rate modulation. The scheme is reported in Fig. 1. $X$ is a cellular resource, whose accumulation controls the metabolic pathways of the cell, definitely regulating in a positive fashion the cell growth rate. The growth rate controls, in turn, the accumulation of a set of metabolic enzymes, by properly tuning both enzymatic production and dilution. In our model $Y$ denotes the class of such enzymes. In summary, $X$ exerts an incoherent feedforward control on $Y$, since it indirectly (by means of the growth rate) enhances both $Y$ production and clearance (feedforward loops are known to be frequently occurring in transcription networks [3], [19]). On the other hand, $Y$ is supposed to control in feedback the $X$ production rate, according to its specific enzymatic affinity. Noise is supposed to affect the expression of $Y$, and the aim of the paper is to characterize how noise propagates on the growth rate from the fluctuations affecting the enzymatic expression. It will be shown that the proposed model (though elementary and basic) is able to replicate important features related to noise propagation through the metabolic pathway, and to support the hypothesis that fluctuations in the enzyme production perturb cellular growth, and not vice versa, because of an apparent positive delay in the cross-correlation function. These results are coherent with recent single-cell experimental results [23], and motivates the research.

A Stochastic Hybrid System (SHS) model is introduced, with the noisy $Y$ production rate modeled in a bursty discrete stochastic fashion, whilst the remaining dynamics evolves according to an Ordinary Differential Equation (ODE) system between any two bursts [10]. SHS models, entailing both continuous and discrete events, are usually preferred (with respect to a more general Chemical Master Equations framework where the chemical reaction network is totally modeled by discrete stochastic events) when the bursty production is dominant with respect to the other reactions, for 


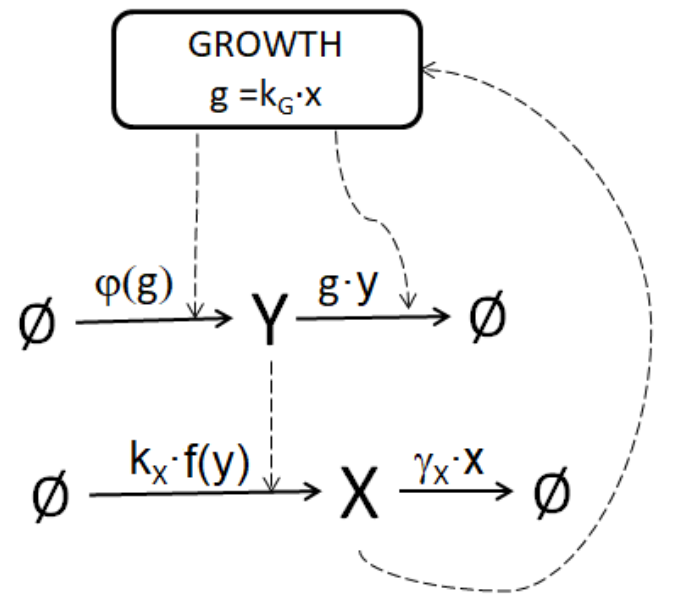

Fig. 1. Schematic figure of the model under investigation.

instance because of a large average burst size [18]. SHS have recently been exploited to investigate the role of feedbacks in noise propagation in enzymatic reaction and metabolic frameworks [4], [5].

Noise propagation is investigated by means of first- and second-order moments, whose analytic computation is, however, prevented by the nonlinearities involved in the model [10], [17], [21]. To overcome such a drawback, analytical solutions are obtained after linearization of the nonlinear functions involved, and these results are validated by the Gillespie Stochastic Simulation Algorithm (SSA) [7], run according to the original nonlinear system, whose results are shown to be very close to the approximated ones on a wide range of model parameters. The SSA is the Monte Carlo numerical approach usually exploited for Chemical Master Equations, since it provides accurate estimates of the probability distributions associated to the chemical reaction network. In the present case, the propensity of the discrete stochastic reaction of the SHS will be shown to depend on the state of the ODE system associated to the SHS, therefore it varies in continuous time. As a consequence, the tau-leaping method [8] will be exploited for stochastic simulation. This is a popular approximate version of SSA, iteratively performing reactions within sufficiently small time steps where propensities are assumed to be approximately constant.

The note is organized as follows. Next Section formally defines the model under study and the kind of investigation that will be carried out. Section III refers to the first-order moment results associated to the SHS, with a special focus on steady-state conditions and stability issues, whilst Section IV details about noise propagation, dealing with second-order moment results, including the cross-correlation analysis. Section $\mathrm{V}$ reports numerical simulations. Conclusions follow.

\section{Model SETTING}

Consider the scheme of Fig. 1 where $X$ and $Y$ refer to the molecular players under investigation and $\mathbf{x}$ and $\mathbf{y}$ refer to their copy numbers. $X$ stands for a cellular resource whose accumulation modulates the growth rate by properly influencing the cell metabolism. By assuming that $X$ is a suitable fraction of the whole cell size, we set the dependency of the growth rate $g$ in terms of a linear function:

$$
g(\mathbf{x})=k_{G} \mathbf{x} .
$$

Indeed, a great deal of experimental results highlights the tight correlation between growth rate and cell size, occurring for different and diverse living cells, like bacteria, fission or budding yeast and mammalian cells (see [2] and references therein).

The growth rate controls, in turn, the accumulation of a set of metabolic enzymes, represented in this model by $Y$, by properly tuning both enzymatic production and dilution. Indeed, dilution is supposed to occur at the same growth rate whilst, regards to $Y$ production, we assume it occurs in bursts of $\eta$ copy numbers, with the random variable $\eta$ indicating the size of the bursts, occurring according to a chosen probability distribution $\mathbb{P}(\eta=j), j=0,1, \ldots$, [20], [9]. The average size of $\eta$ will be shortely denoted by $\bar{\eta}$. Moreover, the propensity $a_{j}$ of an event of $j$ bursts production (i.e. $\mathbf{y} \mapsto \mathbf{y}+j$ ) is assumed to depend on the growth rate via a nonlinear saturating function $\varphi(g)$, chosen to be monotonically increasing (e.g. a saturating exponential or a Michaelis-Menten, according to the experimental results in [12]). Therefore:

$$
a_{j}(\mathbf{x})=\varphi_{g}(\mathbf{x}) \mathbb{P}(\eta=j), \quad j=0,1, \ldots
$$

where

$$
\varphi_{g}(\mathbf{x})=\varphi(g(\mathbf{x}))=\varphi\left(k_{G} \mathbf{x}\right)
$$

In summary, $X$ exerts an incoherent feedforward control on $Y$, since it indirectly (by means of the growth rate) enhances both $Y$ production and clearance.

On the other hand, $Y$ is supposed to control in feedback the resource $X$ production rate, according to a generic monotonically increasing, saturating function of $\mathbf{y}$, denoted by $f(\mathbf{y})$, for instance a Michaelis-Menten function, according to usual assumptions on enzymes kinetics.

According to the Stochastic Hybrid System (SHS) modeling approach [10], denoting with $(\mathbf{x}, \mathbf{y})$ the state of the system associated to the biological framework under investigation, we assume a continuous-time evolution between any two discrete noisy resets, described by the following Ordinary Differential Equation system:

$$
\left\{\begin{array}{l}
\dot{\mathbf{x}}=k_{X} f(\mathbf{y})-\gamma_{X} \mathbf{x} \\
\dot{\mathbf{y}}=-k_{G} \mathbf{x y}
\end{array}\right.
$$

The aim of the work is to investigate how the noise affecting the enzyme production propagates and affects the growth rate. To this end, the cross-correlation function will be exploited, analytically achieved according to the SHS model, by means of first- and second-order moments.

In the following, we will denote with $\langle h(\mathbf{x})\rangle$ the average value of a generic function $h$ of a random process $\mathbf{x}$, and with $\overline{\langle h(\mathbf{x})\rangle}=\lim _{t \mapsto+\infty}\langle h(\mathbf{x})\rangle$ its stationary average value. In case of the average value (i.e. $h(\mathbf{x})=\mathbf{x}$ ), it will be denoted by $\overline{\mathbf{x}}=\overline{\langle\mathbf{x}\rangle}$ for short. 


\section{FIRST-ORDER MOMENTS AND STEADY-STATE CONDITIONS}

The nonlinearities involved in the enzyme clearance rate, in the propensities $a_{j}(\mathbf{x})$ and in the enzymatic feedback on $X$ production rate, prevent from achieving moment equations in closed form. Therefore, we linearize them around the stationary average values $\overline{\mathbf{x}}, \overline{\mathbf{y}}$, supposed to exist and be unique:

$$
\begin{gathered}
\varphi_{g}(\mathbf{x}) \simeq \varphi_{g}(\overline{\mathbf{x}})+\varphi_{g}^{\prime}(\overline{\mathbf{x}})(\mathbf{x}-\overline{\mathbf{x}}) \\
\mathbf{x y} \simeq \overline{\mathbf{x}} \overline{\mathbf{y}}+\overline{\mathbf{y}}(\mathbf{x}-\overline{\mathbf{x}})+\overline{\mathbf{x}}(\mathbf{y}-\overline{\mathbf{y}}) \\
f(\mathbf{y}) \simeq f(\overline{\mathbf{y}})+f^{\prime}(\overline{\mathbf{y}})(\mathbf{y}-\overline{\mathbf{y}})
\end{gathered}
$$

By formally denoting with $\dot{\mathbf{z}}=h(\mathbf{z}), \mathbf{z}=\left[\begin{array}{ll}\mathbf{x} & \mathbf{y}\end{array}\right]^{T}$ the ODE system (4) associated to the SHS, the general formula to achieve any order moment equation from the SHS is below reported [10]

$$
\frac{d}{d t}\langle\psi(\mathbf{z})\rangle=\frac{d \psi}{d \mathbf{z}} h(\mathbf{z})+\sum_{j=1}^{\infty}\left\langle\left(\psi\left(\mathbf{z}+\Delta_{j}\right)-\psi(\mathbf{z})\right) a_{j}(\mathbf{x})\right\rangle
$$

where $\Delta_{j}=\left[\begin{array}{ll}0 & j\end{array}\right]^{T}$ stands for the state update occurring whenever $j$ copies of enzyme $Y$ are produced in bursts with propensity $a_{j}(\mathbf{x})$. Then, first-order moments are readily computed for $\psi(\mathbf{z})=\mathbf{x}$ and $\psi(\mathbf{z})=\mathbf{y}$, by properly exploiting the linear approximations in (5)-(7):

$$
\begin{aligned}
& \frac{d}{d t}\langle\mathbf{x}\rangle=\left\langle k_{X} f(\mathbf{y})-\gamma_{X} \mathbf{x}\right\rangle \\
&= k_{X}\left(f(\overline{\mathbf{y}})+f^{\prime}(\overline{\mathbf{y}})(\langle\mathbf{y}\rangle-\overline{\mathbf{y}})\right)-\gamma_{X}\langle\mathbf{x}\rangle \\
& \frac{d}{d t}\langle\mathbf{y}\rangle=-\left\langle k_{G} \mathbf{x y}\right\rangle+\sum_{j=1}^{\infty}\left\langle j \varphi_{g}(\mathbf{x}) \mathbb{P}(\eta=j)\right\rangle \\
&=-k_{G}(\overline{\mathbf{x}} \overline{\mathbf{y}}+\overline{\mathbf{y}}(\langle\mathbf{x}\rangle-\overline{\mathbf{x}})+\overline{\mathbf{x}}(\langle\mathbf{y}\rangle-\overline{\mathbf{y}})) \\
&+\bar{\eta}\left(\varphi_{g}(\overline{\mathbf{x}})+\varphi_{g}^{\prime}(\overline{\mathbf{x}})(\langle\mathbf{x}\rangle-\overline{\mathbf{x}})\right)
\end{aligned}
$$

First-order moment equations in (9)-(10) can be put in a more compact form according to the following linear system

$$
\frac{d}{d t}\langle\mathbf{z}(t)\rangle=A(\overline{\mathbf{x}}, \overline{\mathbf{y}})\langle\mathbf{z}(t)\rangle+b(\overline{\mathbf{x}}, \overline{\mathbf{y}})
$$

with

$$
\begin{gathered}
A(\overline{\mathbf{x}}, \overline{\mathbf{y}})=\left[\begin{array}{cc}
-\gamma_{X} & k_{X} f^{\prime}(\overline{\mathbf{y}}) \\
\bar{\eta} \varphi_{g}^{\prime}(\overline{\mathbf{x}})-k_{G} \overline{\mathbf{y}} & -k_{G} \overline{\mathbf{x}}
\end{array}\right] \\
b(\overline{\mathbf{x}}, \overline{\mathbf{y}})=\left[\begin{array}{c}
k_{X}\left(f(\overline{\mathbf{y}})-f^{\prime}(\overline{\mathbf{y}}) \overline{\mathbf{y}}\right) \\
\bar{\eta} \varphi_{g}(\overline{\mathbf{x}})+\left(k_{G} \overline{\mathbf{y}}-\bar{\eta} \varphi_{g}^{\prime}(\overline{\mathbf{x}})\right) \overline{\mathbf{x}}
\end{array}\right],
\end{gathered}
$$

providing at steady-state the following algebraic equations:

$$
\left\{\begin{array}{l}
k_{X} f(\overline{\mathbf{y}})=\gamma_{X} \overline{\mathbf{x}} \\
k_{G} \overline{\mathbf{x}} \overline{\mathbf{y}}=\bar{\eta} \varphi_{g}(\overline{\mathbf{x}})
\end{array}\right.
$$

The following Lemma provides a sufficient condition for the existence and uniqueness of a nontrivial, positive solution to the algebraic equations reported in (14).
Lemma 1: Consider the algebraic equation system (14). If $\varphi_{g}(\overline{\mathbf{x}}) / \overline{\mathbf{x}}$ is a monotonically decreasing function in $\overline{\mathbf{x}} \geq 0$, with

$$
\lim _{\overline{\mathbf{x}} \mapsto 0^{+}} \frac{\varphi_{g}(\overline{\mathbf{x}})}{\overline{\mathbf{x}}}=M>0,
$$

then there exists a unique nontrivial, positive solution for the pair $(\overline{\mathbf{x}}, \overline{\mathbf{y}})$.

Proof. According to simple manipulations, it comes that nontrivial positive solutions of (14) are the positive roots of the following nonlinear equation

$$
\Phi(\overline{\mathbf{x}})=0, \quad \Phi(\overline{\mathbf{x}})=k_{X} f\left(\frac{\bar{\eta}}{k_{G}} \cdot \frac{\varphi_{g}(\overline{\mathbf{x}})}{\overline{\mathbf{x}}}\right)-\gamma_{X} \overline{\mathbf{x}},
$$

with

$$
\Phi(0)=k_{X} f\left(\frac{\bar{\eta} M}{k_{G}}\right), \quad \lim _{\overline{\mathbf{x}} \mapsto+\infty} \Phi(\overline{\mathbf{x}})=-\infty,
$$

and

$$
\Phi^{\prime}(\overline{\mathbf{x}})=\frac{k_{X} \eta}{k_{G}} f^{\prime}\left(\frac{\bar{\eta}}{k_{G}} \cdot \frac{\varphi_{g}(\overline{\mathbf{x}})}{\overline{\mathbf{x}}}\right) \frac{d}{d \overline{\mathbf{x}}}\left[\frac{\varphi_{g}(\overline{\mathbf{x}})}{\overline{\mathbf{x}}}\right]-\gamma_{X}<0
$$

because $\varphi_{g}(\overline{\mathbf{x}}) / \overline{\mathbf{x}}$ is monotonically decreasing by hypothesis (15) and $f(\cdot)$ is a monotonically increasing function. Therefore, by continuity, there must exist a unique positive solution for $\overline{\mathrm{x}}$.

In order to investigate the stability of the equilibrium point, we need to show that matrix (12) is Hurwitz, i.e. has eigenvalues with negative real part. The following Lemma provides a sufficient condition.

Lemma 2: Assume that the hypotheses on $\varphi_{g}(\cdot)$ detailed in Lemma 1 hold true and, moreover, that $f(\overline{\mathbf{y}}) / \overline{\mathbf{y}}$ is a monotonically decreasing function for $\overline{\mathbf{y}} \geq 0$. Then, matrix $A$ in (12) is Hurwitz.

Proof. Consider the characteristic polynomial $d(\lambda)$ associated to $A$ :

$$
d(\lambda)=\lambda^{2}+\left(k_{X} \overline{\mathbf{x}}+\gamma_{X}\right) \lambda+c(\overline{\mathbf{x}}, \overline{\mathbf{y}})
$$

with

$$
c(\overline{\mathbf{x}}, \overline{\mathbf{y}})=\gamma_{X} k_{G} \overline{\mathbf{x}}-k_{X} \bar{\eta} f^{\prime}(\overline{\mathbf{y}}) \varphi_{g}^{\prime}(\overline{\mathbf{x}})+k_{X} k_{G} \overline{\mathbf{y}} f^{\prime}(\overline{\mathbf{y}}) .
$$

According to the Routh-Hurwitz criterion, the characteristic polynomial has both roots with negative real part (therefore matrix $A$ is Hurwitz) if, and only if, the zero-order coefficient $c>0$. According to the steady-state conditions (14), after computations, coefficient $c$ can be lower bounded as:

$$
c(\overline{\mathbf{x}}, \overline{\mathbf{y}}) \geq k_{X} \bar{\eta}\left(\frac{f(\overline{\mathbf{y}})}{\overline{\mathbf{y}}} \cdot \frac{\varphi_{g}(\overline{\mathbf{x}})}{\overline{\mathbf{x}}}-f^{\prime}(\overline{\mathbf{y}}) \cdot \varphi_{g}^{\prime}(\overline{\mathbf{x}})\right) .
$$

Now, if $\varphi_{g}(\overline{\mathbf{x}}) / \overline{\mathbf{x}}$ is a decreasing function in $\overline{\mathbf{x}}>0$, then

$$
\begin{aligned}
\frac{d}{d \overline{\mathbf{x}}}\left[\frac{\varphi_{g}(\overline{\mathbf{x}})}{\overline{\mathbf{x}}]}\right]=\frac{\varphi_{g}^{\prime}(\overline{\mathbf{x}}) \overline{\mathbf{x}}-\varphi_{g}(\overline{\mathbf{x}})}{\overline{\mathbf{x}}^{2}}<0 \\
\Longrightarrow \frac{\varphi_{g}(\overline{\mathbf{x}})}{\overline{\mathbf{x}}}>\varphi_{g}^{\prime}(\overline{\mathbf{x}})
\end{aligned}
$$

and, similarly,

$$
\frac{f(\overline{\mathbf{y}})}{\overline{\mathbf{y}}}>f^{\prime}(\overline{\mathbf{y}})
$$

therefore $c(\overline{\mathbf{x}}, \overline{\mathbf{y}})>0$. 
Remark 3: It is worth noticing that the hypotheses of both Lemmas are not restrictive since, for instance, are satisfied by any Michaelis-Menten function of the type

$$
\varphi_{g}(\overline{\mathbf{x}})=k_{Y} \frac{\overline{\mathbf{x}}}{\overline{\mathbf{x}}+\theta_{y}},
$$

as well as by exponentials of the form

$$
\varphi_{g}(\overline{\mathbf{x}})=k_{Y}\left(1-e^{-\theta_{y} \overline{\mathbf{x}}}\right) .
$$

\section{SECOND-ORDER MOMENTS AND CROSS-CORRELATION FUNCTION}

To deal with noise fluctuations and propagation, we need to compute the second-order moments associated to the copy number of the species of the system. The dynamic equations for $\left\langle\mathbf{x}^{2}\right\rangle,\left\langle\mathbf{y}^{2}\right\rangle,\langle\mathbf{x y}\rangle$ can be written according to (8) by properly setting $\psi(\mathbf{z})=\mathbf{x}^{2}, \psi(\mathbf{z})=\mathbf{y}^{2}$ and $\psi(\mathbf{z})=\mathbf{x y}$. Because of the linearization, the steady-states solutions $\overline{\left\langle x^{2}\right\rangle}$, $\overline{\left\langle\mathbf{y}^{2}\right\rangle}, \overline{\langle\mathbf{x y}\rangle}$ are obtained as the solutions of the following linear third-order system

$$
\begin{gathered}
\gamma_{X} \overline{\left\langle\mathbf{x}^{2}\right\rangle}-k_{X} f^{\prime}(\overline{\mathbf{y}}) \overline{\langle\mathbf{x y}\rangle}=k_{X} \overline{\mathbf{x}} \overline{\mathbf{y}}\left(\frac{f(\overline{\mathbf{y}})}{\overline{\mathbf{x}}}-f^{\prime}(\overline{\mathbf{y}})\right) \\
k_{G} \overline{\mathbf{x}} \overline{\left\langle\mathbf{y}^{2}\right\rangle}+\bar{\eta}\left(\frac{\varphi_{g}(\overline{\mathbf{x}})}{\overline{\mathbf{x}}}-\varphi_{g}^{\prime}(\overline{\mathbf{x}})\right) \overline{\langle\mathbf{x y}\rangle} \\
=\frac{\left\langle\eta^{2}\right\rangle}{2} \varphi_{g}(\overline{\mathbf{x}})+\overline{\mathbf{x}} \overline{\mathbf{y}} \bar{\eta}\left(2 \frac{\varphi_{g}(\overline{\mathbf{x}})}{\overline{\mathbf{x}}}-\varphi_{g}^{\prime}(\overline{\mathbf{x}})\right) \\
k_{X} \overline{\left\langle\mathbf{y}^{2}\right\rangle}-\bar{\eta}\left(\frac{\varphi_{g}(\overline{\mathbf{x}})}{\overline{\mathbf{x}}}-\varphi_{g}^{\prime}(\overline{\mathbf{x}})\right) \overline{\left\langle\mathbf{x}^{2}\right\rangle}-\left(\gamma_{X}+k_{G} \overline{\mathbf{x}}\right) \overline{\langle\mathbf{x y}\rangle} \\
=-\overline{\mathbf{x}} \overline{\mathbf{y}}\left(\gamma_{X}+k_{G} \overline{\mathbf{x}}\right)+k_{X} \overline{\mathbf{y}}^{2} f^{\prime}(\overline{\mathbf{y}})-k_{G} \overline{\mathbf{x}}^{2} \overline{\mathbf{y}}+\bar{\eta} \overline{\mathbf{x}}^{2} \varphi_{g}^{\prime}(\overline{\mathbf{x}}) \\
=-k_{X} \overline{\mathbf{y}}^{2}\left(\frac{f(\overline{\mathbf{y}})}{\overline{\mathbf{y}}}-f^{\prime}(\overline{\mathbf{y}})\right)-\bar{\eta} \overline{\mathbf{x}}^{2}\left(2 \frac{\varphi_{g}(\overline{\mathbf{x}})}{\overline{\mathbf{x}}}-\varphi_{g}^{\prime}(\overline{\mathbf{x}})\right)
\end{gathered}
$$

The explicit solution is readily achievable, though its final form is cumbersome and not easy-to-handle, therefore it will not be reported here.

These results on stationary second-order moments will be exploited to compute the cross-correlation function, in order to infer information on whether noise fluctuations in the enzyme production propagate on cellular growth, or vice versa. With a little abuse of notation, whenever the explicit dependency of time is required, the following notation is exploited for short:

$$
g(\mathbf{x}(t))=g(t)
$$

so that the cross-correlation coefficient between $\mathbf{y}$ and $g$ is formally defined as

$$
\rho_{y g}(\tau)=\frac{\overline{\langle\mathbf{y}(t) g(t+\tau)\rangle}-\overline{\mathbf{y}} g(\overline{\mathbf{x}})}{\sigma_{Y} \sigma_{g}},
$$

where $\tau \in \mathbb{R}$ is the lag, describing a delay related to noise propagation, and $\sigma_{Y}, \sigma_{g}$ are the stationary standard deviations associated to $g$ and $Y$, respectively, with

$$
\sigma_{g}^{2}=\overline{\left\langle(g(\mathbf{x})-g(\overline{\mathbf{x}}))^{2}\right\rangle}=\overline{\left\langle k_{G}^{2}(\mathbf{x}-\overline{\mathbf{x}})^{2}\right\rangle}=k_{G}^{2} \sigma_{X}^{2} .
$$

In summary:

$$
\rho_{y g}(\tau)=\frac{\overline{\langle\mathbf{y}(t) \mathbf{x}(t+\tau)\rangle}-\overline{\mathbf{x y}}}{\sigma_{X} \sigma_{Y}}=\rho_{y x}(\tau) .
$$

For positive lags $\tau \geq 0$, similarly to [16], we first compute the cross-covariance by properly exploiting the relationship

$$
\langle\mathbf{y}(t) \mathbf{x}(t+\tau)\rangle=\langle\mathbf{y}(t)\langle\mathbf{x}(t+\tau) \mid \mathbf{x}(t), \mathbf{y}(t)\rangle\rangle .
$$

Instead, for negative lags, we need to resort to another conditional expectation formula. Indeed, for $\tau<0$

$$
\begin{aligned}
& \langle\mathbf{y}(t) \mathbf{x}(t+\tau)\rangle=\langle\mathbf{y}(t) \mathbf{x}(t-|\tau|)\rangle \\
& \quad=\langle\mathbf{x}(t-|\tau|)\langle\mathbf{y}(t) \mid \mathbf{x}(t-|\tau|), \mathbf{y}(t-|\tau|)\rangle\rangle
\end{aligned}
$$

and so, at steady-state:

$$
\overline{\langle\mathbf{y}(t) \mathbf{x}(t+\tau)\rangle}=\overline{\langle\mathbf{x}(t) \mathbf{y}(t+|\tau|)\rangle} .
$$

Let $\tau \geq 0$. Then, by defining $C_{x}=\left[\begin{array}{ll}1 & 0\end{array}\right], C_{y}=\left[\begin{array}{ll}0 & 1\end{array}\right]$ and according to (11), we have:

$$
\langle\mathbf{x}(t+\tau) \mid \mathbf{x}(t), \mathbf{y}(t)\rangle=C_{x} e^{A \tau} \mathbf{z}(t)+C_{x} \int_{t}^{t+\tau} e^{A(t+\tau-s)} b d s
$$

that becomes

$$
\langle\mathbf{x}(t+\tau) \mid \mathbf{x}(t), \mathbf{y}(t)\rangle=C_{x} e^{A \tau} \mathbf{z}(t)+C_{x} A^{-1}\left(e^{A \tau}-I\right) b
$$

provided that $A$ is nonsingular. By substituting (37) into (33) we have

$$
\begin{aligned}
\langle\mathbf{y}(t) \mathbf{x}(t+\tau)\rangle=C_{x} e^{A \tau}\langle & \mathbf{y}(t) \mathbf{z}(t)\rangle \\
& +\langle\mathbf{y}(t)\rangle C_{x} A^{-1}\left(e^{A \tau}-I\right) b
\end{aligned}
$$

and so, accounting for steady-state solutions, when $t \mapsto+\infty$ we have

$$
\overline{\langle\mathbf{y}(t) \mathbf{x}(t+\tau)\rangle}=C_{x} e^{A \tau} \overline{\langle\mathbf{y z}\rangle}+\overline{\mathbf{y}} C_{x} A^{-1}\left(e^{A \tau}-I\right) b
$$

with

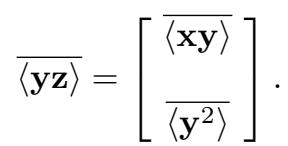

Analogously, let $\tau<0$. Then, by means of (35) we compute

$$
\langle\mathbf{x}(t) \mathbf{y}(t+|\tau|)\rangle=\langle\mathbf{x}(t)\langle\mathbf{y}(t+|\tau|) \mid \mathbf{x}(t), \mathbf{y}(t)\rangle\rangle .
$$

Then, according to (11), we have:

$$
\begin{aligned}
\langle\mathbf{y}(t+|\tau|) \mid \mathbf{x}(t), \mathbf{y}(t)\rangle & =C_{y} e^{A|\tau|} \mathbf{z}(t) \\
+ & C_{y} \int_{t}^{t+|\tau|} e^{A(t+|\tau|-s)} b d s
\end{aligned}
$$

that becomes

$\langle\mathbf{y}(t+|\tau|) \mid \mathbf{x}(t), \mathbf{y}(t)\rangle=C_{y} e^{A|\tau|} \mathbf{z}(t)+C_{y} A^{-1}\left(e^{A|\tau|}-I\right) b$

provided that $A$ is nonsingular. By substituting (43) into (41) we have

$$
\begin{aligned}
\langle\mathbf{x}(t) \mathbf{y}(t+|\tau|)\rangle=C_{y} e^{A|\tau|} & \langle\mathbf{x}(t) \mathbf{z}(t)\rangle \\
& +\langle\mathbf{x}(t)\rangle C_{y} A^{-1}\left(e^{A|\tau|}-I\right) b
\end{aligned}
$$


TABLE I

NOMINAL MODEL PARAMETERS.

\begin{tabular}{|c|c|}
\hline Parameter & Value \\
\hline$\lambda$ & 0.2 \\
\hline$k_{X}$ & 1000 \\
\hline$\theta_{F}$ & 300 \\
\hline$\gamma_{X}$ & 1 \\
\hline$\theta_{y}$ & 500 \\
\hline$k_{G}$ & $2 \cdot 10^{-4}$ \\
\hline$k_{Y}$ & 15 \\
\hline
\end{tabular}

and so, accounting for steady-state solutions, when $t \mapsto+\infty$ we have

$$
\overline{\langle\mathbf{x}(t) \mathbf{y}(t+|\tau|)\rangle}=C_{y} e^{A|\tau|} \overline{\langle\mathbf{x z}\rangle}+\overline{\mathbf{x}} C_{y} A^{-1}\left(e^{A|\tau|}-I\right) b
$$

with

$$
\overline{\langle\mathbf{x z}\rangle}=\left[\begin{array}{l}
\overline{\left\langle\mathbf{x}^{2}\right\rangle} \\
\overline{\langle\mathbf{x y}\rangle}
\end{array}\right] .
$$

\section{NUMERICAL SIMULATIONS}

Numerical simulations are carried out according to the following choice for the nonlinear functions $f(\cdot)$ and $\varphi(\cdot)$. Both these functions are set as Michaelis-Menten functions, so that conditions in Lemmas 1-2 are trivially satisfied

$$
f(\mathbf{y})=\frac{\mathbf{y}}{\mathbf{y}+\theta_{F}}, \quad \varphi(g)=k_{\phi} \frac{g}{g+\theta_{\phi}} .
$$

Because of the linear relationship between $\mathbf{x}$ and $g$, the choice of $\varphi(g)$ in (47) implies that also $\varphi_{g}(\mathbf{x})$ is a MichaelisMenten of the type (24), with $k_{Y}=k_{\phi} \theta_{y}=\theta_{\phi} / k_{G}$. Regards to the bursty noisy production of $Y$, similarly to [20], [4], we assume the following geometric probability distribution:

$$
\mathbb{P}(\eta=j)=(1-\lambda)^{j} \lambda, \quad \lambda \in(0,1], \quad j=0,1, \ldots
$$

providing an average burst size $\bar{\eta}=(1-\lambda) / \lambda$.

A set of nominal model parameters is reported in Table I, according to which the steady-state solutions provided by (14) are $\overline{\mathbf{x}}=500, \overline{\mathbf{y}}=300$. Notice that nominal parameters have been set in order to provide steady-state values equal to the Michaelis-Menten constants (i.e. $\overline{\mathbf{x}}=\theta_{y}$ and $\overline{\mathbf{y}}=\theta_{F}$ ), thus providing half of the maximum values for $\varphi_{g}(\overline{\mathbf{x}})=$ $k_{Y} / 2$ and $f(\overline{\mathbf{y}})=1 / 2$, respectively.

Stochastic simulations for the SHS are performed by means of the tau-leaping algorithm [8] to validate the theoretical approximate results developed in the previous sections, by also exploiting the ergodic properties of the underlying stochastic process. In this setting, the step selection has been chosen equal to 0.1 seconds within an overall simulation time of 10,000 seconds.

Figs. 2-3 illustrate the SHS equilibrium distributions of species $X$ and $Y$ obtained via tau-leaping, which result to be centered on the equilibria $\overline{\mathbf{x}}$ and $\overline{\mathbf{y}}$ of (14), respectively.

In order to compare cross-correlation functions according to different model parameter settings, we assume to vary the Michaelis-Menten parameters $\theta_{y}$ and $\theta_{F}$ of the feedback functions as well as the values $k_{Y}$ and $k_{X}$ in order to keep

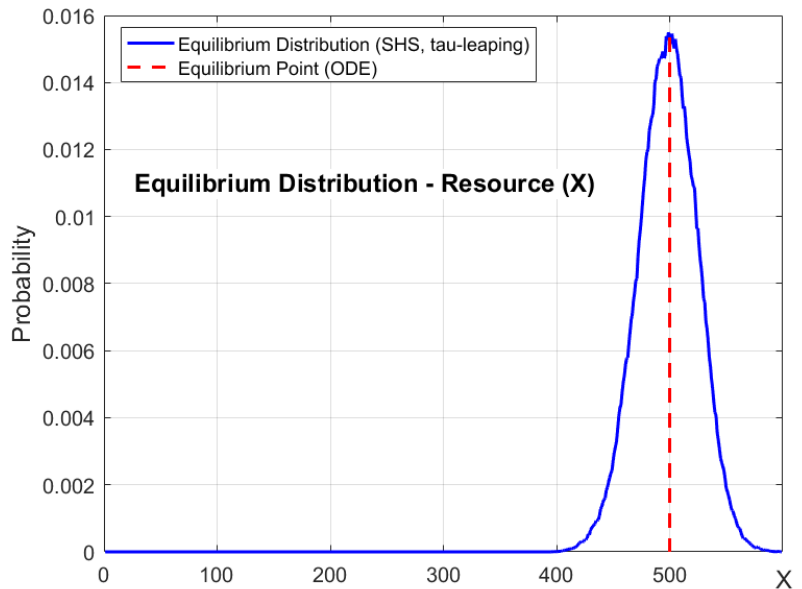

Fig. 2. Statistical distribution of species $X$ of the SHS (2)-(4) obtained by means of the $\tau$-leaping method (blue solid line). The mode of the distribution is approximately centered on the equilibrium solution $\overline{\mathbf{x}}$ of (14) (red dashed line).

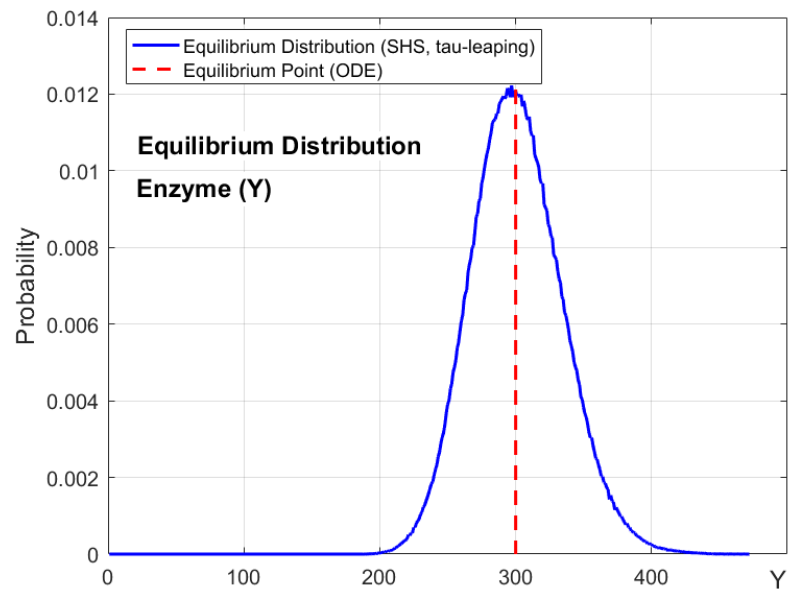

Fig. 3. Statistical distribution of species $Y$ of the SHS (2)-(4) obtained by means of the $\tau$-leaping method (blue solid line). The mode of the distribution is approximately centered on the equilibrium value $\overline{\mathbf{y}}$ of (14) (red dashed line).

fixed the steady-state solutions achieved for the nominal values. In other words, by denoting with $M M_{f}, M M_{\phi}$ the following constants,

$$
M M_{f}=k_{X} \frac{\overline{\mathbf{y}}}{\overline{\mathbf{y}}+\theta_{F}}, \quad M M_{\phi}=k_{Y} \frac{\overline{\mathbf{x}}}{\overline{\mathbf{x}}+\theta_{y}}
$$

equations (49) provide the loci on the planes $\left(k_{X}, \theta_{F}\right)$ and $\left(k_{Y}, \theta_{y}\right)$, respectively, where to take values compatible with the fixed steady-state solutions.

Cross-correlation functions have been computed by arbitrarily varying parameters $\theta_{F}$ and $\theta_{y}$ and by varying parameters $k_{X}$ and $k_{Y}$ according to (49). Table II reports the kind of variation together with the measurement of the feedback strength on $f(\cdot)$ and $\varphi_{g}(\cdot)$ computed as

$$
S_{f}=k_{X} \cdot \frac{f^{\prime}(\overline{\mathbf{y}})}{\overline{\mathbf{y}}}, \quad S_{\phi}=\frac{\varphi_{g}^{\prime}(\overline{\mathbf{x}})}{\overline{\mathbf{x}}} .
$$


TABLE II

PARAMETER VARIATIONS AND FEEDBACK STRENGTH.

\begin{tabular}{|c|c|c|}
\hline Parameter Variation & $S_{f}$ & $S_{\phi}$ \\
\hline$\theta_{F}, \theta_{y}$ & 0.0028 & $1.5 \cdot 10^{-5}$ \\
\hline $10 \cdot \theta_{F}, \theta_{y}$ & 0.0051 & $1.5 \cdot 10^{-5}$ \\
\hline $0.1 \cdot \theta_{F}, \theta_{y}$ & $5.1 \cdot 10^{-4}$ & $1.5 \cdot 10^{-5}$ \\
\hline$\theta_{F}, 10 \cdot \theta_{y}$ & 0.0028 & $2.7 \cdot 10^{-5}$ \\
\hline$\theta_{F}, 0.1 \cdot \theta_{y}$ & 0.0028 & $2.7 \cdot 10^{-6}$ \\
\hline
\end{tabular}

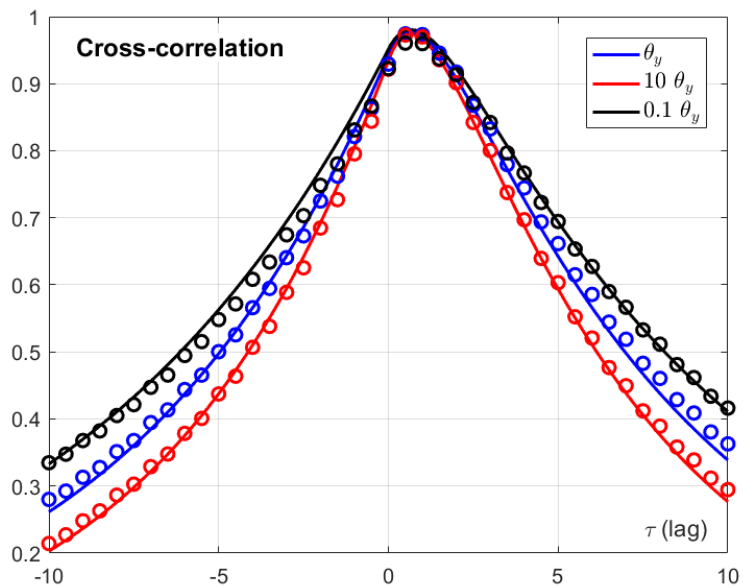

Fig. 4. Cross-correlation function $\rho_{y g}(\tau)$ drawn according to different values of parameter $\theta_{y}$. The analytical computations (solid lines) are approximately validated by the statistical results obtained by means of the $\tau$-leaping method (circles).

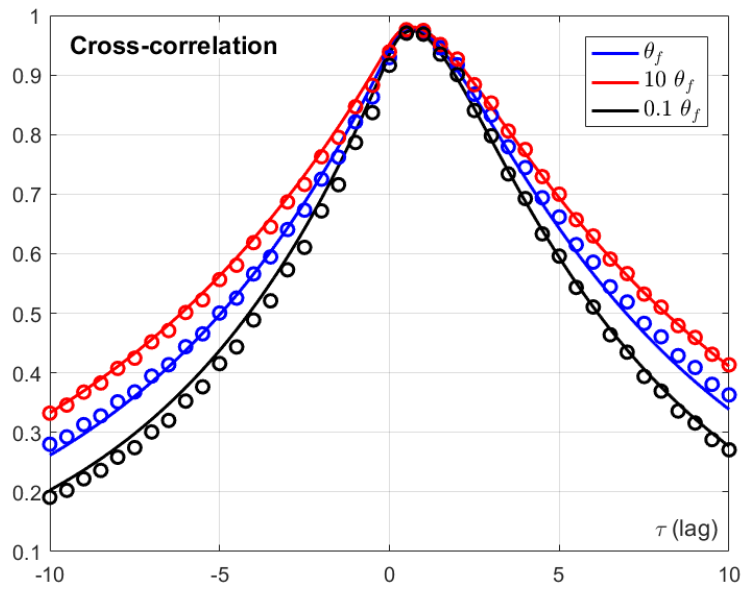

Fig. 5. Cross-correlation function $\rho_{y g}(\tau)$ drawn according to different values of parameter $\theta_{F}$. The analytical computations (solid lines) are approximately validated by the statistical results obtained by means of the $\tau$-leaping method (circles).
Figs. 4-5 draw the cross-correlations according to the aforementioned scheme, where approximate formulae are again validated by tau-leaping stochastic simulations, with same simulation step (0.1 seconds) and time horizon (10, 000 seconds) for each parameter set in Table II. The nominal case is reported in blue in both figures. One apparent fact is that the maximum of the cross-correlation function occurs in correspondence of a positive delay. This fact is coherent with the analogous experimental results reported in [23], where it was highlighted that current enzyme expression correlates better with growth some time later, thus explaining that enzyme production fluctuations happen first and growth fluctuations occur some time later. In other words, growth fluctuations arise because of the noise in the enzyme expression, and not vice versa. Another apparent fact that is shared by all parameter settings is that the maximum correlation lag does not move if we keep fixed the steady-state solutions. On the other hand, by increasing the strength of the enzymatic $(Y)$ feedback on the resource $(X)$ (i.e. by increasing $S_{f}$ ), we have an amplification of the cross-correlation function, and the opposite happens if we decrease $S_{f}$, see Fig. 5 and Table II: that means the feedback of $Y$ on $X$ production rate enhances the correlation between the expression of the metabolic enzyme and cellular growth rate. Instead, with respect to the feedforward of $X$ accumulation on the enzyme production rate, an increase on the feedforward strength $S_{\phi}$ weakens the cross-correlation function (and vice versa when $S_{\phi}$ decreases): in summary, the feedback of $Y$ on $X$ and the feedforward of $X$ on $Y$ provide opposite effects on the cross-correlation function.

\section{CONCLUSiOnS}

This work investigates a simple model of interplay among growth rate, metabolism and resource allocation, in presence of noise affecting the enzymatic activity. Approximate moment computations and cross-correlation functions based on a Stochastic Hybrid System framework are validated via approximate stochastic simulations, in order to evaluate how noise propagates in the metabolic pathway. Consistently with recent experiments, the hypothesis that fluctuations in the enzyme production perturb cellular growth (and not viceversa), are corroborated because of an apparent delay appearing in the cross-correlation function. Future work will be devoted to investigate the impact of a second source of noise, allowing parameter $k_{X}$ in $X$ production rate to be stochastic.

\section{ACKNOWLEDGEMENTS}

PP is supported by the MIUR grant SysBioNet Italian Roadmap for ESFRI Research Infrastructures, SYSBIO Centre of Systems Biology, Milan and Rome, Italy. AS was supported by the National Science Foundation Grant ECCS 1711548

\section{REFERENCES}

[1] L. Alberghina, G. Mavelli, G. Drovandi, P. Palumbo, S. Pessina, F. Tripodi, P. Coccetti, M. Vanoni, Cell growth and cell cycle in Saccharomyces cerevisiae: Basic regulatory design and proteinprotein interaction network, Biotechnol. Adv., 30, 52-72, 2012. 
[2] M. Aldea, K. Jenkins, A. Csikász-Nagy, Growth Rate as a Direct Regulator of the Start Network to Set Cell Size, Front. Cell Dev. Biol., 5:57, 2017

[3] U. Alon, An Introduction to Systems Biology: Design Principles of Biological Circuits, Chapman and Hall/CRC, 2006.

[4] A. Borri, P. Palumbo, A. Singh, Impact of negative feedback in metabolic noise propagation, IET Syst. Biol., 1-8, 2016.

[5] A. Borri, P. Palumbo, A. Singh, Noise propagation in a class of metabolic networks, Proceedings of the 56th IEEE Conference on Decision and Control (CDC 2017), Melbourne, Australia, 447-452, 2017.

[6] M. Cook, M. Tyers, Size control goes global, Curr. Opin. Biotechnol. 18, 341350, 2007

[7] D. T. Gillespie, Exact Stochastic Simulation of Coupled Chemical Reactions, The Journal of Physical Chemistry 81(25), 23402361, 1977.

[8] D. T. Gillespie, Approximate accelerated stochastic simulation of chemically reacting systems, The Journal of Chemical Physics 115(4): 1716, 2001.

[9] I. Golding, J. Paulsson, S. Zawilski, E. Cox, Real-time kinetics of gene activity in individual bacteria, Cell 123, 1025-1036, 2005.

[10] J.P. Hespanha, A. Singh, Stochastic models for chemically reacting systems using polynomial stochastic hybrid systems, Int. J. of Robust and Nonlinear Control, 15, 669-689, 2005.

[11] D.J. Kiviet, P. Nghe, N. Walker, S. Boulineau, V. Sunderlikova, S.J. Tans, Stochasticity of metabolism and growth at the single-cell level. Nature, 514, 376-379, 2014.

[12] S. Klumpp, Z. Zhang, T. Hwa, Growth Rate-Dependent Global Effects on Gene Expression in Bacteria, Cell, Volume 139, Issue 7, pp. 1366$1375,2009$.

[13] A. Lloyd, The regulation of cell size, Cell, 154, 11941205, 2013
[14] W.F. Marshall, K.D. Young, M. Swaffer, E. Wood, P. Nurse, A Kimura, et al., What determines cell size? BMC Biol. 10:101, 2012

[15] N. Nordholt, J. van Heerden, R. Kort, F.J. Bruggeman, Effects of growth rate and promoter activity on single-cell protein expression, Scientific Reports, 7:6299, DOI:10.1038/s41598-017-05871-3, 2018

[16] A. Singh, B. Bokes, Consequences of mRNA Transport on Stochastic Variability in Protein Levels, Biophysical Journal 103, 1087-1096, 2012.

[17] A. Singh, J.P. Hespanha, Approximate moment dynamics for chemically reacting systems, IEEE Transactions on Automatic Control. 56, 414-418, 2011.

[18] M. Soltani, P. Bokes, Z. Fox, A. Singh, Nonspecific transcription factor binding can reduce noise in the expression of downstream proteins, Phys. Biol., 12, p.055002, 2015.

[19] M. Soltani, T. Platini, A. Singh, Stochastic analysis of an incoherent feedforward genetic motif, proceedings of the American Control Conference (ACC 2016), Boston, MA, pp. 406-411, 2016.

[20] M. Soltani, C. Vargas, A. Singh, Conditional moment closure schemes for studying stochastic dynamics of genetic circuits. IEEE Transactions on Biomedical Circuits and Systems, 9(4), 518-526, 2015.

[21] E. D. Sontag and A. Singh, Exact Moment Dynamics for Feedforward Nonlinear Chemical Reaction Networks, in IEEE Life Sciences Letters, 1(2), 26-29, 2015.

[22] N.G. van Kampen, Stochastic Processes in Physics and Chemistry, North Holland, third edition, 2007.

[23] M. Wehrens, F. Buke, P. Nghe, S.J. Tans, Stochasticity in cellular metabolism and growth: Approaches and consequences, Current Opinion in Systems Biology, 8, 131-136, 2018 